\title{
Estimación de la demanda y el costo de no tener agua potable en Nicaragua, tomando como base las diferentes alternativas de abastecimiento (puestos, ríos, manantiales, pipas, carretas y

Carlos Narváez ${ }^{1}$ y Carlos Meza²

\footnotetext{
${ }^{1}$ Profesor horario, Departamento de Economía Aplicada, Universidad Centroamericana. Rotonda Rubén Darío 150 mts. al oeste. Apartado 69, Managua, Nicaragua. Correo electrónico: carlnarsil@yahoo.com

2 Miembro de la Red de Expertos Iberoamericanos en Energía y funcionario de la Gerencia Comercial de ALBA GENERACIÓN. Managua, Nicaragua. Correo electrónico:mezagonzalez_8@yahoo.com
}

Recibido: enero de 2010 / Aceptado: junio de 2010

EL ESTUDIO ESTIMA LA DEMANDA DE AGUA DE LAS VIVIENDAS que no cuentan con el servicio de agua potable por medio de tubería. La estimación se realiza utilizando la Encuesta de Medición de Nivel de Vida (INIDE, 2005a), donde se presenta información sobre las formas de abastecimiento de agua, el tiempo dedicado a la recolección, el costo monetario por galón, entre otras variables socioeconómicas. Se eligió el modelo teórico basado en el enfoque de producción de hogares propuesto por Bockstael y McConnell (1983) que permite estimar el excedente del consumidor como medida de beneficios potenciales del hogar por contar con agua potable dentro de sus viviendas.

Los beneficios estimados se calcularon tomando en cuenta el costo de oportunidad en el tiempo dado a un proceso de búsqueda de agua por parte del hogar más el pago por el bien en las diferentes alternativas (puestos, ríos, manantiales, pipas, carretas y pozos).

La investigación mira desde otra perspectiva el modelo de costo de viaje usado comúnmente en estudios de recreación ambiental y pone a disposición de los evaluadores de proyectos una herramienta alternativa de la estimación de beneficios en proyectos de abastecimiento de agua. 
Las personas sin acceso al agua pagan 316 córdobas por mes (C\$/Mes) y consumen aproximadamente 2 metros cúbicos mensuales. Un cliente con servicio de agua por medio de la red de ENACAL con un consumo de 15 metros cúbicos paga por cargo de agua potable $72.75 \mathrm{C} \$ / \mathrm{Mes}$. Es importante mencionar que existen otros cargos como alcantarillado sanitario y cargo fijo que deben sumarse al cargo de agua potable en una factura correspondiente a viviendas en los departamentos en donde el problema de falta de agua es mayor. Por tanto, el mismo consumo (15 $\left.\mathrm{M}^{3} / \mathrm{Mes}\right)$ por vivienda -si incluimos todos los cargos- pagaría $98.75 \mathrm{C} \$ / \mathrm{Mes}$. El cargo de agua potable para consumos de $30 \mathrm{M}^{3} / \mathrm{Mes}$ es igual a $168 \mathrm{C} \$$, una cifra mucho menor a lo que pagan las personas que no cuenta con el servicio. Los datos obtenidos se basan en el pliego tarifario actual.

Palabras clave: demanda de agua / modelo lin-log / costo de viaje /excedente del consumidor

\section{Introducción}

El acceso al agua en muchos países representa un problema agudo. El Programa de Naciones Unidas para el Desarrollo enfatiza que en muchos países de Latinoamérica existe una abrumadora inequidad entre la población de altos y bajos ingresos, rurales y urbanos, al acceso del servicio de agua potable de calidad.

La población más afectada por la falta de agua potable son los niños. Se ha estimado que cerca de un millón 800 mil niños mueren cada año a causa de enfermedades gastrointestinales, lo que significa un promedio de 4,900 muertes diarias. El número de muertos por diarrea en el año 2004 fue seis veces mayor a la media de mortalidad anual de los conflictos armados en la década de los 90 (Jouravlev, 2008).

Además, es importante destacar que aproximadamente la mitad de la población de países en desarrollo sufren de alguna enfermedad provocada por la falta de agua potable y cada año los niños pierden 443 millones de días escolares debido a enfermedades relacionada a la falta de suministro de agua potable (Jouravlev, 2008).

Nicaragua es conocida como tierra de lagos y volcanes, tiene un potencial y reserva de agua en los dos grandes lagos: Cocibolca y Xolotlán. Sin embargo, a pesar de que existe una gran abundancia del recurso, muchos hogares nicaragüenses tienen dificultad para acceder a agua potable.

De acuerdo al Censo 2005 (Instituto Nacional de Información de Desarrollo [INIDE], 2005b), Nicaragua tiene una población de 6 millones de personas y aproximadamente un millón de viviendas, de las cuales el $49 \%$ no cuenta con suministro de agua por medio de tubería, siendo los más afectados la zona rural y los pobres extremos.

En base a lo anterior, podemos decir que la falta de agua potable es uno de los principales problemas de la población. Ante este problema vale la pena preguntarse: ¿cuánto representa 
en términos monetarios el excedente del consumidor de los hogares por no tener agua potable dentro de su vivienda? ¿cuál es la demanda de agua en metros cúbicos de las viviendas que no tienen el servicio de agua?

Por medio del modelo de costo de viaje, ajustados a una forma funcional lineal-logarítmica, se estima el excedente del consumidor tomando como base el costo de oportunidad por no tener agua y el costo monetario.

El artículo se encuentra organizado en ocho secciones. En la sección 2 se presenta la situación actual al acceso del agua en Nicaragua, en la sección 3 está la revisión de estudios similares a nuestra investigación, las secciones 4,5 y 6 presentan el modelo teórico, empírico y los datos utilizados. Finalmente se presentan los resultados, conclusiones y recomendaciones (secciones 7 y 8).

\section{Situación actual del acceso al agua}

El acceso al agua en Nicaragua sigue siendo un problema latente. En 2001 el 44\% de las viviendas no contaba con el suministro de agua por medio de tubería ya sea dentro de la vivienda como fuera pero dentro del terreno. Para el año 2005, el 49\% de las viviendas tenían que abastecerse de las diferentes alternativas (puesto público, pozo público o privado, ojo de agua o manantial, río, quebrada, arroyo, lago, lagunas, pipas, etc.). El 80\% de los pobres extremos no accede al consumo de agua por medio de la red de la Empresa Nicaragüense de Acueductos y Alcantarillados (ENACAL). Es importante destacar que municipios con fuentes hídricas tienen problemas de abastecimiento de agua, tal es el caso de los municipios dentro de la cuenca del Lago Cocibolca.

En diciembre de 2007, la cantidad de clientes de ENACAL era de 435,596 para una cobertura del 43\% (ENACAL, 2007 y 2008) si tomamos en cuenta el total de viviendas reportadas en el Censo 2005 (1 millón). Para diciembre de 2008, la empresa de agua facturaba a 475,089 clientes, teniendo un incremento de 39,493 clientes. Sin embargo, este incremento no se debe necesariamente a servicios nuevos ya que involucra los clientes legalizados. Si se toma como referencia la cantidad de clientes facturados en diciembre de 2008 la cobertura de abastecimientos es del 48\%, un dato similar al obtenido en 2005 por la Encuesta de Medición de Nivel de Vida [EMNV] (INIDE, 2005a). Por tanto, en base a los datos presentados podemos afirmar que aún persiste la dificultad de acceder al servicio de agua por medio de la red de ENACAL.

Ilustración 1. Consumos medios en $\mathrm{M}^{3}$ por departamentos (zona del pacífico)

Consumo medios (M3) por viviendas en los departamentos

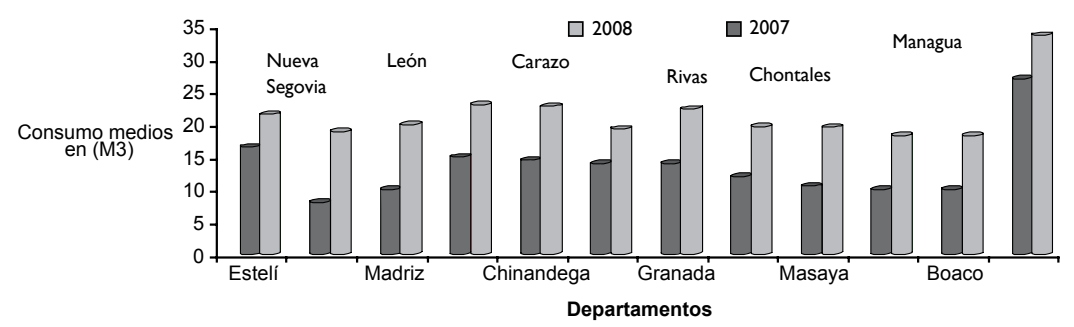

Fuente: Autores en base a estadísticas de ENACAL, 2007 y 2008 
En 2008 los consumos medios en $\mathrm{M}^{3}$ por vivienda aumentaron significativamente. Por ejemplo, Managua (con los consumos medios más altos para 2008) tuvo un incremento del $25 \%$ respecto al 2007, pero en departamentos como Nueva Segovia, Boaco, Chontales y Masaya los incrementos fueron mayores al $70 \%$.

\section{Ilustración 2. Mapa de consumo y producción $\left(\mathrm{M}^{3}\right)$ de agua en Nicaragua}
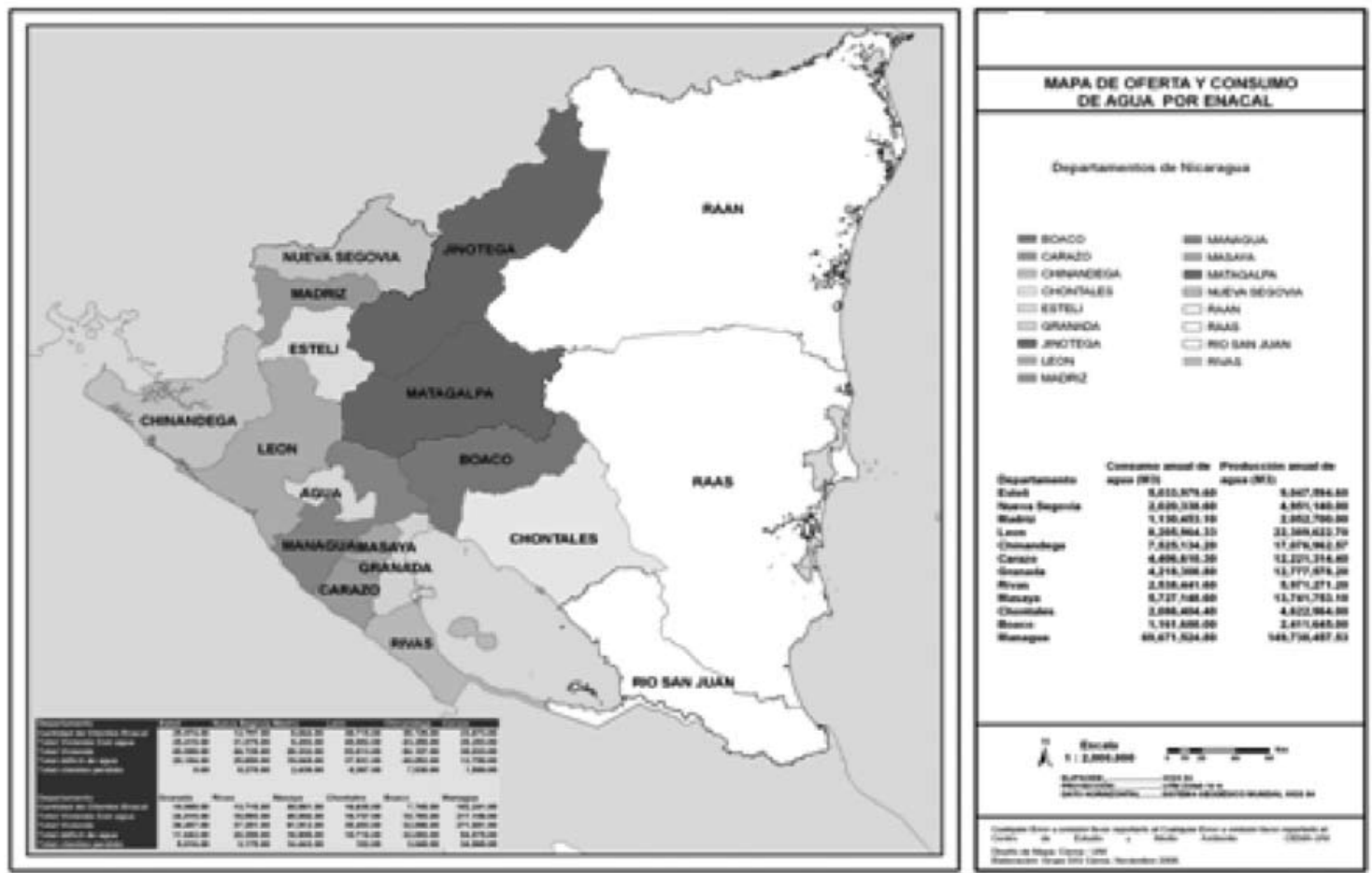

Fuente: Autores en base a datos Enacal (2007 y 2008)

Como se puede apreciar en la ilustración 2, la producción de agua $\left(\mathrm{M}^{3}\right)$ potable en todos los departamentos supera en más del $40 \%$ la facturación $\left(\mathrm{M}^{3}\right)$, siendo la oferta de agua suficiente para satisfacer a la cantidad de clientes. El problema se centra en las pérdidas de agua, tanto de clientes ilegales como de tuberías obsoletas.

En el censo 2005 (INIDE, 2005b), la cantidad de viviendas con tuberías era de 595,700. Una de las formas que existe actualmente (por la ausencia de registros estadísticos y la no inversión) para determinar la cantidad de clientes ilegales y dar una aproximación de la cantidad de consumo no facturado es: restando las 595,700 viviendas (Censo 2005) menos la cantidad de clientes de ENACAL a diciembre de 2008, igual a 475,089, lo que da un resultado de 120,611 (ver cuadro 1). De acuerdo a la ilustración 2 los departamentos en rojo no forman parte de los clientes de ENACAL, por lo que es necesario restárselo a los 120,611. El resultado proporciona unas 55 mil viviendas ilegales. Si asumimos el consumo medio más alto, por ejemplo $33 \mathrm{M}^{3} /$ Vivienda, la cantidad de agua no facturada sería de aproximadamente 22 millones de $\mathrm{M}^{3} / \mathrm{año}$. 
Cuadro 1. Comparación de viviendas con agua potable ENACAL y Censo 2005

\begin{tabular}{|c|c|c|c|}
\hline \multicolumn{2}{|c|}{ Censo 2005 } & ENACAL 2008 \\
\cline { 1 - 2 } Clientes con tubería & \multirow{2}{*}{ Clientes } \\
\cline { 1 - 2 } Total clientes con tubería & Matagalpa & Jinotega & \\
\hline 595,700 & 26,118 & 9,094 & 475,089 \\
\hline
\end{tabular}

Fuente: Autores en base a datos del Censo 2005 (INIDE, 2005b) y facturación de ENACAL (2007 y 2008)

A continuación presentamos una tabla comparativa con los principales indicadores de empresas de agua.

Cuadro 2. Indicadores

\begin{tabular}{|l|l|l|}
\hline \multicolumn{1}{|c|}{ Santiago de Chile } & \multicolumn{1}{c|}{ Buenos Aires } & \multicolumn{1}{c|}{ Nicaragua } \\
\hline Alto nivel de cobertura & Bajo nivel de cobertura & Bajo nivel de cobertura \\
\hline *Agua potable $100 \%$ & *Agua potable $71 \%$ & $*$ Agua potable $50 \%$ \\
\hline *Alcantarillado $97 \%$ & *Alcantarillado $57 \%$ & *Alcantarillado $40 \%$ \\
\hline Alto nivel de eficiencia & Bajo nivel de eficiencia & Bajo nivel de eficiencia \\
\hline *Pérdidas de agua: $25 \%$ & $*$ Pérdidas de agua: $45 \%$ & *Pérdidas de agua: $55 \%$ \\
\hline $\begin{array}{l}\text { *Empleados/mil conexiones } \\
3\end{array}$ & $\begin{array}{l}\text { *Empleados/mil conexiones } \\
8\end{array}$ & *Empleados/mil conexiones \\
\hline *Consumo: 210 Lt/hab/día & *Consumo: 500 Lt/hab/día & *Consumo: 75 Lt/hab/día \\
\hline Alta micro-medición:100\% & Baja micro-medición: $15 \%$ & Baja micro-medición: $59 \%$ \\
\hline Desarrollo e inversión & Colapso & Colapso \\
\hline
\end{tabular}

Fuente: Autores en base a estadísticas 2007 (ENACAL, 2007 y 2008) y CEPAL (Jouravlev, 2008)

El 15\% de las pérdidas de agua son causadas por los "ilegales" en Nicaragua. Por tanto, si se mejora la eficiencia, el porcentaje de agua no contabilizada sería menor al 50\%. El problema es aún mayor y un gran desafío para la actual administración ya que el agua no contabilizada incluye a clientes sin medidor. Éstos representaban en 2007 el 41\% (184, 427) del total de clientes en ese año (448, 283 clientes). A este tipo de cliente se le factura una cuota fija que oscila en rangos de $\left(20-26 \mathrm{M}^{3}\right)$ por vivienda. La ilustración 1 muestra consumos medios mayores a $30 \mathrm{M}^{3}$. 
Ilustración 3. Consumo de agua, demanda óptima y oferta.

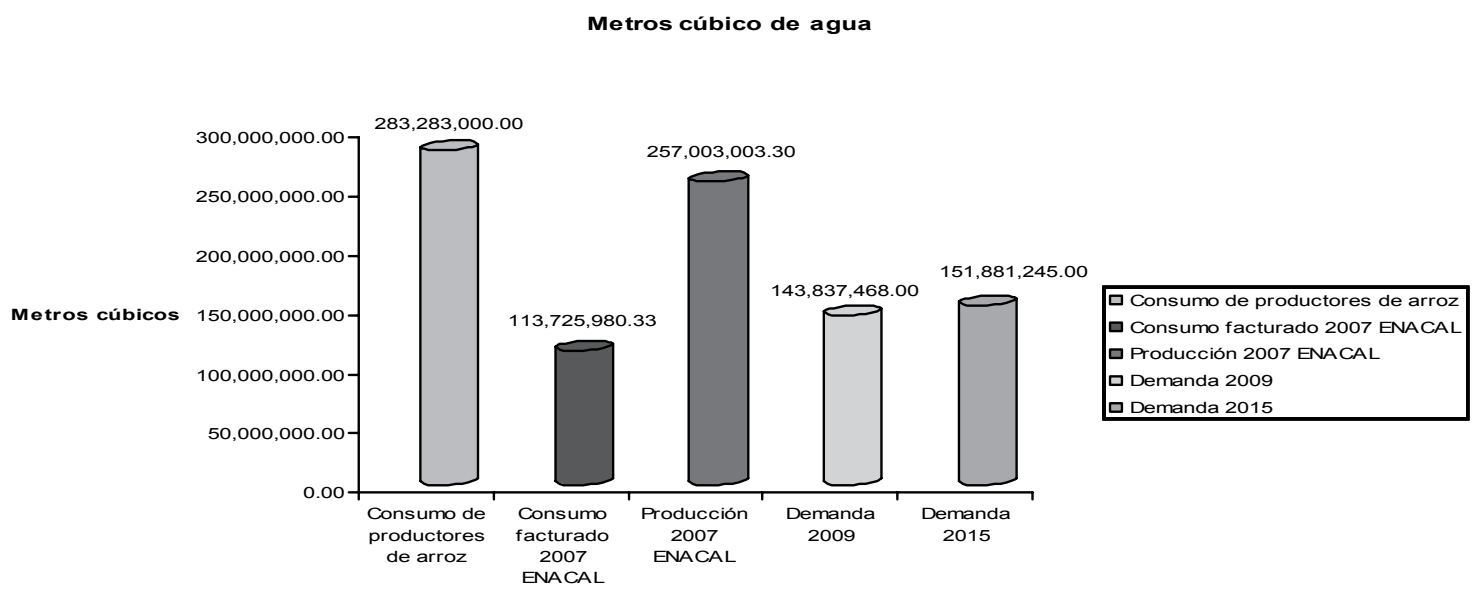

Fuente: Autores en base al Censo Agropecuario (INIDE, 2001), Censo de Población (INIDE, 2005b) y ENACAL (2007 y 2008)

En la ilustración 3 se observa que se produce suficiente agua para satisfacer la demanda óptima al 2015. Sin embargo, los productores de arroz consumen el doble de lo que demandará la población al 2015. Esto se debe a que no existe una regulación vía precios por el agua que consumen y probablemente no hagan uso racional. De igual manera, es necesario estimar el consumo en las industrias con pozos propios y establecer un canon por utilizar el bien agua como insumo en sus procesos productivos.

\section{Revisión de literatura}

Existen muchos estudios en los que se ha evaluado la demanda de las diferentes alternativas de consumo de agua. Seleccionamos algunas investigaciones que han utilizado la aplicación empírica de los modelos económicos con el objetivo de hacer una mejor interpretación de los resultados de la investigación. 


\begin{tabular}{|c|c|c|}
\hline $\begin{array}{c}\text { Autor } \\
\text { Año }\end{array}$ & Título & Contenido principal \\
\hline 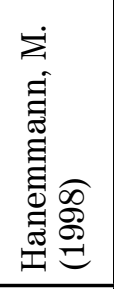 & 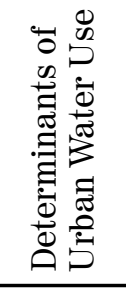 & $\begin{array}{l}\text { La investigación consiste en determinar la demanda de agua en dos campos, } \\
\text { el uso del agua industrial y el uso de agua residencial. Para el último toma en } \\
\text { cuenta dos puntos de vista: el uso por unidad familiar, el uso multifamilia. En } \\
\text { el marco teórico toma en cuenta una función de utilidad de los consumidores } \\
\text { usando diferentes formas funcionales: Cobb-Douglas y Stone Geary. El análisis } \\
\text { usando esas funciones lleva a determinar las elasticidades y concluye que el } \\
\text { agua es un bien normal en ambos casos }\end{array}$ \\
\hline 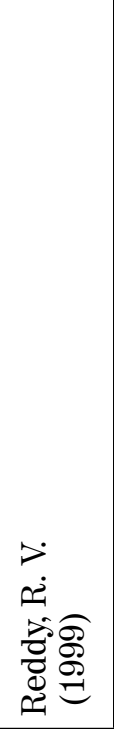 & 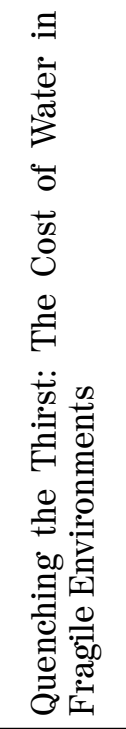 & $\begin{array}{l}\text { Estudia el costo del agua en la India (Rajasthan state). Se basa principalmente } \\
\text { en información de seis pueblos sobre la escasez del agua, el ingreso de las } \\
\text { familias, tamaño del hogar, precio del agua y la distancia que existe de la casa } \\
\text { a la fuente. } \\
\text { Examina los diferentes costos de acuerdo al uso del agua. Además estima la } \\
\text { disponibilidad a pagar por el agua usando el método de valoración contingente. } \\
\text { En términos de política concluye que: (i) la estimación de la demanda de agua } \\
\text { ayudará a diseñar los sistemas de oferta en las zonas más sensibles de la } \\
\text { región de una manera pragmática, (ii) debe ser la principal prioridad para } \\
\text { los gobiernos proveer este servicio a los hogares en las zonas rurales con un } \\
\text { abastecimiento regular ya que bajaría el costo de oportunidad en el tiempo } \\
\text { que cada hogar invierte en ir a la fuente por agua, especialmente las mujeres } \\
\text { y niños, (iii) es un error asumir que los hogares pueden pagar el } 5 \% \text { de su } \\
\text { ingreso per cápita. El estudio indica que la disponibilidad a pagar por tener el } \\
\text { servicio de agua potable en su hogar representa solamente el } 2 \% \text { de su ingreso } \\
\text { per cápita en el área rural. En nuestro trabajo se estima el excedente del } \\
\text { consumidor tanto para la zona rural y urbana usando el modelo de costo de } \\
\text { viaje y con información de jefes de hogares. }\end{array}$ \\
\hline 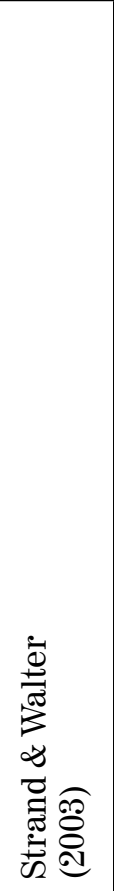 & 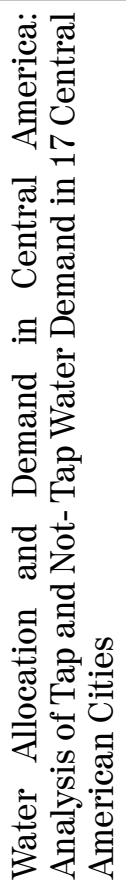 & $\begin{array}{l}\text { La investigación se refiere a la asignación y demanda de agua con y sin } \\
\text { servicio por medio de tubería en las viviendas. El estudio se realizó en } 17 \text { sitios } \\
\text { de Centro América e incluye a Venezuela. Los datos utilizados comprenden } \\
11,500 \text { viviendas. La encuesta proporciona datos de tres tipos: viviendas con } \\
\text { servicio de agua por tubería y con medidores, viviendas con servicio de agua } \\
\text { sin medidor y hogares sin servicios de agua que utilizan formas alternativas } \\
\text { de abastecimiento. } \\
\text { El estimador de la demanda de agua para las viviendas con tuberías y } \\
\text { medidas (medidor), tomando como base los datos, es difícil de encontrar } \\
\text { debido a que utilizan tarifas por bloques de consumo (ver en anexo } 1 \text { pliego } \\
\text { tarifario de Nicaragua), en tal sentido que el precio marginal del agua para } \\
\text { una vivienda por lo general tiene un incremento por consumo. La relación } \\
\text { marginal y promedio entre el precio del agua y el consumo es positiva, lo } \\
\text { que se espera que para la función de demanda la relación sea negativa para } \\
\text { establecer políticas de precios que regulen el consumo. En el estudio, para } \\
\text { corregir la relación directa entre el consumo y el precio se aplican variables } \\
\text { instrumentales. Así mismo, los modelos más adecuados para este tipo de } \\
\text { estudios son las relaciones lineales-logarítmicas. Una vez estimada la función } \\
\text { de demanda de agua por vivienda analizan las elasticidades y concluyen que } \\
\text { las viviendas cuando no tiene el servicio de agua por tubería consumen } 5.5 \mathrm{M}^{3} \\
\text { contra } 30 \mathrm{M}^{3} \text { y enfrentan precios mucho más altos de entre } 5 \text { versus } 0.4 \mathrm{U} \$ \\
\mathrm{M}^{3} \text { y el tiempo invertido en halar agua de la fuente de abastecimiento a las } \\
\text { viviendas es de aproximadamente } 11 \text { hrs por mes. }\end{array}$ \\
\hline
\end{tabular}


Torrado (2001), en un estudio de estimación de la demanda de agua, presenta un modelo teórico y econométrico. Utiliza el modelo de Burtless y Hausman (1978, citados en Torrado, 2001) aplicado en un modelo de oferta de trabajo que posee una restricción presupuestal no lineal. El autor llega a la conclusión de que la demanda de agua es caracterizada por un grupo de observaciones alrededor de cada rango de consumo. Por tanto, la demanda de agua es claramente multimodal. Así mismo, a partir del modelo se puede determinar las elasticidades sin presentar los inconvenientes que presenta el hacer estimaciones econométricas por mínimos cuadrados ordinarios y de variables instrumentales. Esto permite analizar una política de precios marginal de cada categoría y rangos de consumos.

En Nicaragua son muy pocos los estudios económicos que se han hecho en el área de economía del bienestar. Víquez (2005) presenta una evaluación de la situación de la falta de agua potable en Nicaragua. Un elemento notable es su fuerte marco de economía política en el que se refiere al por qué la población tiene problemas de suministro de agua potable. Concluye que existe una ineficiencia en la asignación del recurso por parte de ENACAL y que la población con problemas de abastecimiento de agua no ejerce ninguna presión a las autoridades encargadas.

Avendaño (2004) estima el valor económico del agua en Nicaragua para consumo doméstico, industrial y de riego. Encontró el valor del agua cruda destinada a consumo doméstico en Managua en C $\$ 0,16 / \mathrm{m}^{3}$, esto es US\$ 0,01 por $\mathrm{m}^{3}$. La desventaja es que la elasticidad fue supuesta y no se logró estimar ninguna curva de demanda para determinar el excedente del consumidor por la falta de información. Además, los datos provienen de las personas con servicio de agua por medio de la red de ENACAL. En nuestra investigación solamente consideramos a las viviendas que no cuentan con el servicio.

\section{Marco teórico}

\subsection{Método de costo de viaje}

El modelo de costo de viaje es un método de valoración de bienes que no tienen un mercado definido y se basa en la extensión de la teoría de la demanda del consumidor, en la cual el valor del tiempo es la principal atención. El que inició los estudios en este campo fue el economista Harold Hotelling, quien en 1974 aplicó por primera vez el modelo con el propósito de estimar los beneficios por recreación de un parque natural en Estados Unidos.

La premisa que rige el modelo es que el tiempo tiene un valor económico. La idea consiste en utilizar información relacionada con la cantidad de tiempo utilizada, la cual implica un costo de oportunidad y de dinero que representa el gasto real que una persona realiza con la finalidad de llegar a un sitio con el objetivo de recrearse y disfrutarlo (Romero, 1997). En la presente investigación aplicamos la metodología pero no con desarrollo de actividades de recreación o turísticas de un sitio natural en especial, sino más bien con el desplazamiento de un sitio de origen como la vivienda del hogar a un sitio de destino representado por el lugar donde se encuentran las formas de abastecimiento de agua en Nicaragua.

La economía del bienestar aplicado nos proporciona las herramientas necesarias para poder determinar los beneficios de una política o proyectos de abastecimiento de agua 
usando diferentes enfoques. No obstante, las investigaciones serán dedicadas a extender diversas formas de aplicar una metodología que lleve a una mejor estimación y por ende a valorar de una mejor manera los servicios de agua como un bien público.

El trabajo se centra en poder valorar la pérdida de bienestar en términos de costo monetario que produce la falta del servicio de agua dentro de las viviendas ya que las personas tienen que incurrir en viajes en busca del abastecimiento (puesto público, pozo público o privado, ojo de agua o manantial, río, quebrada, arroyo, camión, carreta o pipa, lago, laguna vecinos u otros).

Los objetivos, supuestos, estrategias y características de la metodología del costo de viaje y la obtención de la función de demandas Marshallianas se tomaron en cuenta de acuerdo al manual de valoración económica de bienes no mercadeables (Mendieta \& Caraballo, 2005).

Supuestos de la metodología:

- La persona encargada de ir por el agua maximiza su bienestar sujeta a la restricción de ingreso y de tiempo.

- Cada viaje persigue un solo propósito.

- Existe un costo de oportunidad del tiempo.

- Para muchos individuos la demanda por el bien es igual a cero.

- El bien tiene un mercado observable, el sitio donde se abastecen de agua.

- Para consumir el agua, el jefe del hogar debe viajar o transportarse hasta el sitio.

Objetivos:

- Estimar el valor del uso del bien, partiendo del hecho de que sólo una persona realiza el viaje.

- Determinar el valor económico que las familias asignan por el consumo de agua. Estrategias:

- Basarse en el comportamiento de los individuos.

- La información se obtiene por medio de encuestas datos de corte transversal.

- La estimación de la función de demanda de viajes por agua se realizará considerando diferentes formas funcionales.

\subsection{Teoría del consumidor}

Las variables que se utilizan en el modelo:

$q=$ Número de viajes

$c=$ Costo incurrido en desplazarse al sitio $(\mathrm{C} \$)$

$t=$ Tiempo empleado en cada viaje (Min)

$x=$ Pago por agua en el sitio (C\$) 
Supuesto:

- El tiempo tiene un valor y por tanto:

$T=$ Tiempo total disponible

$h=$ Unidades de tiempo del trabajo del individuo asignado

$w=$ Salario

El tiempo total: $T=t+h$

La unidad de tiempo de trabajo será:

$h=T-t$, que es el tiempo empleado en el trabajo. Por tanto, la restricción de tiempo es $T=h+t^{*} q$. Ecuación que nos describe el tiempo que las personas dedican al trabajo y a viajar para recoger agua.

La restricción de presupuesto viene determinada por:

$w=I=c^{*} q+p^{*} x$ ecuación 1

Donde: $c^{*} q=$ Ingreso gastado por desplazarse al sitio $\mathrm{p}^{*} \mathrm{x}=$ Ingreso gastado en pagar la compra de agua

Se puede observar en base a la ecuación 1 que $q$ y $x$ son las únicas opciones en las que se asigna el ingreso. Los hogares que no cuentan con el suministro de agua tienen que realizar viajes por adquirir el bien, en los que se invierte un cierto tiempo y el resto del tiempo quedará libre o disponible para obtener más ingreso. Esto se puede representar mediante la siguiente ecuación:

$q\left(c+x^{*} t\right)-p * x=I$ .ecuación 2

La ecuación 2 representa el costo incurrido en compra de agua más el costo de oportunidad en el tiempo, que será igual a la cantidad de ingreso que obtiene el individuo más su ingreso fijo.

Dadas las ecuaciones 1 y 2 obtenemos una función de demanda de viajes por agua. Para ello se necesita maximizar una función de utilidad sujeta a la restricción o ecuación 2.

Maximizar $U(q, x)$ sujeta a $q\left(c+w^{*} t\right)-p^{*} x=I$, aplicando el método del multiplicador de Lagrange para obtener la demanda Marshalliana para $q$ que es la variable que representa el número de viajes para recoger agua:

$L=U(q, x)+\mathrm{I}\left\{I-q\left(c+w^{*} t\right)+p^{*} x\right\}$ ecuación 3

Aplicando las condiciones de primer orden:

$q=f\left\{\left(c+w^{*} t\right)\right\}$ .ecuación 4 
Gráficamente y por motivo de representación asumiremos lineal la forma funcional de la ecuación 4:

Ilustración 4. Función de demanda de viajes por agua

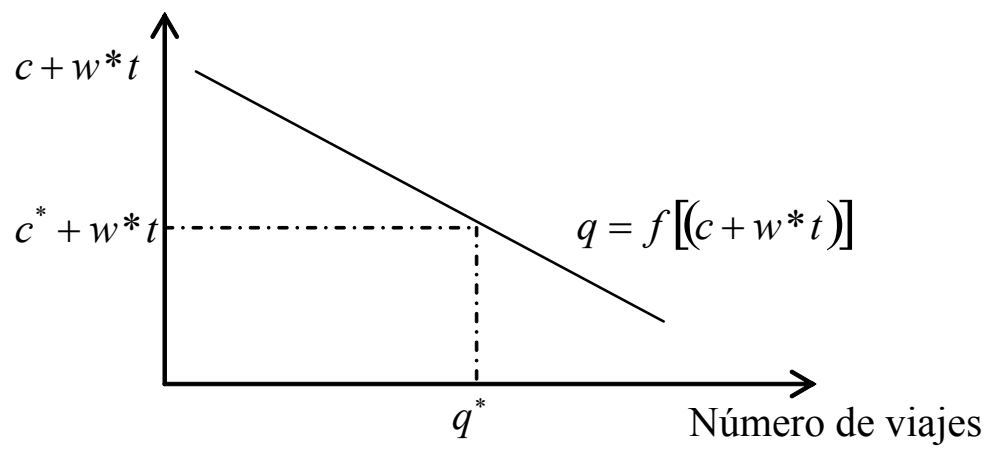

La ilustración 4 muestra que a mayores costos el número de viajes será menor. También representa el área que determina el excedente del consumidor (EC), definido como el área por debajo de la curva de demanda y por encima del costo. Se interpreta como una medida de disponibilidad a pagar por consumir una cantidad del bien y la cantidad que el consumidor realmente paga (Call \& Holahan, 1983).

4.3. El problema del costo de oportunidad en el tiempo

En la investigación utilizaremos el tiempo como un costo de oportunidad que los miembros de las familias realizan por adquirir agua, de manera similar a la que se aplica para valorar recursos naturales de uso recreativo como lagos, playas y otras zonas públicas (Uribe, Mendieta, Jaime \& Carriazo, 2003).

Existen estudios en los que se ha demostrado que el costo de oportunidad en el tiempo tiene un valor sobre el salario comprendido entre el 25\% y 50\% (Smith \& Kaoru, 1990).

Cuadro 3. Forma de estimación del costo de oportunidad

\begin{tabular}{|c|c|}
\hline Salario diario (No pobreza) & Salario diario ( Extrema pobreza) \\
\hline$C O^{D I A}=\frac{\operatorname{Tg}_{\min }^{\text {dia }}[\operatorname{Salario}(1-0.35)]}{T t_{\mathrm{min}}^{d i a}}$ & $C O^{D I A}=\frac{\operatorname{Tg}_{\min }^{\text {dia }}[\operatorname{Salario}(1-0.25)]}{T t_{\min }^{d i a}}$ \\
\hline$T g_{\min }^{\text {dia }}$ Tiempo gastado en el viaje min/día. & $T g_{\min }^{\text {dia }}$ Tiempo gastado en el viaje min/día. \\
\hline$T t_{\min }^{d i a}$ Tiempo total de trabajo min/ día. & $T t_{\min }^{\text {dia }}$ Tiempo total de trabajo min/ día. \\
\hline$T t_{\min }^{d i a}=\frac{60 \mathrm{~min}^{*} 8 h}{1 h}=480 \mathrm{~min} /$ dia & $T t_{\min }^{\text {dia }}=\frac{60 \mathrm{~min} * 8 h}{1 h}=480 \mathrm{~min} /$ dia \\
\hline
\end{tabular}

Fuente: Autores en base a Smith \& Kaoru (1990) 


\section{Datos}

Cuadro 4. Estadísticas descriptivas de las variables utilizadas en el modelo

\begin{tabular}{|c|c|c|c|c|c|}
\hline Variables & \multicolumn{2}{|l|}{ Descripción } & Frecuencia & Promedio & Variable \\
\hline Edad & \multicolumn{2}{|l|}{ Edad reportada del jefe de hogar } & 6830 & 48 & Continua \\
\hline Personas & \multicolumn{2}{|l|}{ Personas por viviendas en Nicaragua } & 6830 & 6 & Continua \\
\hline Hogares & \multicolumn{2}{|l|}{ Cantidad de hogares por viviendas } & 6830 & 1 & Continua \\
\hline $\mathrm{CO}$ & \multicolumn{2}{|c|}{$\begin{array}{l}\text { Costo de oportunidad en el tiempo en } \mathrm{C} \$ / \text { minutos en un } \\
\text { mes }\end{array}$} & 3065 & 11.7 & Continua \\
\hline \multirow{5}{*}{ Educación } & \multirow{5}{*}{ Nivel de Educación } & 0: Ninguno & 1,886 & ----- & \multirow{5}{*}{ Cualitativa } \\
\hline & & 1: Primaria & 1,471 & ----- & \\
\hline & & 2: Secundaria & 509 & ---- & \\
\hline & & 3:Técnico & 94 & ----- & \\
\hline & & 4: Profesional & 433 & ---- & \\
\hline \multirow[t]{2}{*}{ Residencia } & \multirow{2}{*}{ Área } & 1: Urbano & 4,084 & ----- & \multirow{2}{*}{ Dummy } \\
\hline & & 2: Rural & 2,746 & --- & \\
\hline \multirow{2}{*}{ Sexo } & \multirow{2}{*}{ Género } & 1: Hombre & 4,726 & ---- & \multirow[b]{2}{*}{ Dummy } \\
\hline & & 2: Mujer & 2,104 & - & \\
\hline \multirow{9}{*}{ Alternativas } & \multirow{9}{*}{$\begin{array}{l}\text { Abastecimiento de } \\
\text { agua en las viviendas }\end{array}$} & 1: Tubería & 3,765 & ---- & \multirow{9}{*}{ Cualitativa } \\
\hline & & $\begin{array}{ll}\text { 2: } & \text { Puesto } \\
\text { público } & \end{array}$ & 184 & ---- & \\
\hline & & $\begin{array}{l}\text { 3: Pozo público } \\
\text { o privado }\end{array}$ & 1,269 & ---- & \\
\hline & & $\begin{array}{l}\text { 4: Ojo de agua } \\
\text { o manantial }\end{array}$ & 764 & ---- & \\
\hline & & $\begin{array}{l}\text { 5:Río,quebrada, } \\
\text { arroyo }\end{array}$ & 376 & --.-- & \\
\hline & & $\begin{array}{l}\text { 6: Camión, } \\
\text { carreta, pipa }\end{array}$ & 12 & --- & \\
\hline & & 7:Lago, laguna & 8 & - & \\
\hline & & 8: Vecino & 421 & --- & \\
\hline & & 9: Otro & 31 & - - & \\
\hline \multirow{5}{*}{ Consumo } & \multirow{5}{*}{ Formas de consumo } & 1: Metro cúbico & 1893 & ----- & \multirow{5}{*}{ Cualitativa } \\
\hline & & 2: Galones & 247 & --- & \\
\hline & & 3: Bidones & 2,260 & ---- & \\
\hline & & 4: Barriles & 1,106 & ---- & \\
\hline & & 5: No sabe & 1,232 & ---- & \\
\hline \multirow{4}{*}{ Pago } & \multirow{4}{*}{$\begin{array}{l}\text { Pago por agua en un mes antes de la } \\
\text { encuesta en } \mathrm{C} \$\end{array}$} & 1: Metro cúbico & 1864 & 90.50376 & \\
\hline & & 2: Galones & 34 & 32.91176 & \\
\hline & & 3: Bidones & 461 & 25.68547 & \\
\hline & & 4: Barriles & 345 & 53.72464 & \\
\hline
\end{tabular}




\begin{tabular}{|c|c|c|c|c|c|}
\hline \multirow{7}{*}{ Ingreso } & \multirow{7}{*}{ Ultimo ingreso obtenido en C\$ } & 1: Semanal & 1,812 & 464.676 & \multirow{7}{*}{ Cualitativa } \\
\hline & & 2: Catorcenal & 50 & 733.76 & \\
\hline & & 3: Quincenal & 765 & 1261.958 & \\
\hline & & 4: Mensual & 1,439 & $4,801.051$ & \\
\hline & & 5: Trimestral & 50 & 6440.44 & \\
\hline & & 6: Semestral & 476 & 6870.393 & \\
\hline & & 7: Anual & 1,071 & 22703.44 & \\
\hline \multirow{4}{*}{ Frecuencia } & \multirow{4}{*}{ Número de viajes } & 1: Día & 2,980 & 1 & \multirow{4}{*}{ Continua } \\
\hline & & 2: Semana & 476 & 2 & \\
\hline & & 3: Quincena & 15 & 4 & \\
\hline & & 4: Mes & 1,982 & 8 & \\
\hline \multirow{3}{*}{ Distancia } & \multicolumn{2}{|l|}{ Distancia en KM } & 90 & 1.977778 & \multirow{5}{*}{ Continuas } \\
\hline & Distancia en Mt & & 1823 & 129.7043 & \\
\hline & Distancia en Vrs & & 1122 & 94.76916 & \\
\hline \multirow[t]{2}{*}{ Tiempo } & Hrs & & 72 & 2.972222 & \\
\hline & Minutos & & 2953 & 10.40061 & \\
\hline \multirow{3}{*}{ Pobreza } & Pobres extremos & & 1,038 & & \multirow{3}{*}{ Cualitativa } \\
\hline & Pobres & & 2,063 & & \\
\hline & No pobres & & 3,729 & & \\
\hline
\end{tabular}

Fuente: Autores en base a la EMNV 2005 (INIDE, 2005a)

Solamente el 37\% de la población en pobreza extrema accede al agua por medio de tubería. Las fuentes alternativas son: ríos, lagunas y pozos públicos. En la zona rural el problema es más agudo ya que el 87\% de las viviendas no cuenta con abastecimiento de agua potable por medio de la red de ENACAL (INIDE, 2005a).

La variable número de viajes fue creada en base a investigaciones realizadas en otros países y la pregunta de la sección 1, pregunta $29 \mathrm{C}$, en la que aparece la frecuencia del tiempo (día, semana, quincena y mes) junto con la variable formas de consumo (bidones, barriles y galones) presentada en la EMNV 2005 (INIDE, 2005a).

El costo de oportunidad en el tiempo también es una variable desarrollada por los investigadores en base al cuadro 3 y los datos de la EMNV 2005 (INIDE, 2005a).

Cuadro 5. Pagos por consumo de agua mensuales en córdobas (C\$)

\begin{tabular}{|l|c|c|c|c|}
\hline \multirow{2}{*}{ Fuente } & \multicolumn{2}{c|}{ Área } & \multicolumn{2}{c|}{ Pobreza } \\
\cline { 2 - 5 } & Urbano & Rural & Extrema & No extrema \\
\hline Tubería dentro de la vivienda & 102.5062 & 64.04854 & 43 & 70.48171 \\
\hline $\begin{array}{l}\text { Tubería fuera de la vivienda, pero en } \\
\text { el terreno }\end{array}$ & 74.4116 & 42.33155 & 27.71636 & 56.4949 \\
\hline Puesto público & 29.73684 & 38.54167 & 28.47059 & 26.22581 \\
\hline
\end{tabular}




\begin{tabular}{|l|c|c|c|c|}
\hline Pozo público o privado & 41.14815 & 21.42424 & 18.52632 & 22.66667 \\
\hline Ojo de agua o manantial & 0 & 0 & 0 & 0 \\
\hline Río, quebrada, arroyo & 0 & 0 & 0 & 0 \\
\hline Lago, laguna & 119 & 176.5 & 0 & 210 \\
\hline Vecino & 0 & 0 & 0 & 0 \\
\hline Otros & 59.02899 & 36.21429 & 30.58333 & 65.05263 \\
\hline
\end{tabular}

Fuente: Autores en base a la EMNV 2005 (INIDE, 2005a)

Los pagos son muy variables por fuente de abastecimiento de agua. Es importante señalar que los consumos son diferentes cuando no se tiene agua debido a las diferencias en cuanto a las unidades en que se transporta el agua, las cuales pueden ser barriles, bidones y galones. El precio, cuando se tiene agua y son viviendas en pobreza extrema, es aproximadamente de 30 córdobas en promedio por mes. Los pagos por agua de las alternativas pozo, puesto, etc., no incluyen el costo de oportunidad en el tiempo por desplazarse al sitio en donde se encuentra ubicada la fuente.

De acuerdo al Anuario de Estadísticas 2010 del Banco Central de Nicaragua (BCN, 2010), los salarios mensuales son mayores a los reportados en 2005 por el INSS. El promedio fue de 3,437.90C\$. En esta investigación utilizamos el último ingreso obtenido en C\$ por mes (ver cuadro 4 para mayores detalles).

\section{Modelo econométrico}

La forma funcional a la que se ajustaron los datos de las variables en el cuadro 5 es una lineal-logarítmica (lin-log). Los parámetros obtenidos $b_{i}$ fueron estimados por mínimos cuadrados ordinarios. Recuérdese que pueden ser Cobb-Douglas, Binomial Negativa o Poisson en estos casos. Además, se excluyeron las observaciones con tubería ya sea dentro como fuera de la vivienda. Es importante mencionar que la utilización de un modelo lin-log es conveniente porque los datos tienen una naturaleza discreta, la cual implica que existe un número de viajes al sitio $(1,2,3 \ldots)$ en un tiempo determinado. Así mismo existen personas que no realizan viajes por tanto es igual a cero.

Cuadro 6. Descripción de las variables

\begin{tabular}{|l|l|l|l|}
\hline Variable & Definición & Coeficiente & Signo esperado \\
\hline$\beta_{i}$ & Parámetros en el modelo & ------- & \\
\hline NSA & Número de viajes por servicio de agua & ---------------- \\
\hline & $\begin{array}{l}\text { Constante del intercepto con el eje del costo } \\
\text { total }\end{array}$ & $\beta_{0}^{*}$ & - ----------------- \\
\hline---------------- \\
\hline CT & $\begin{array}{l}\text { Costo de viaje por recoger agua, incluye el } \\
\text { precio que se paga por las diferentes formas } \\
\text { de recolección y el costo de oportunidad en } \\
\text { el tiempo en C\$ }\end{array}$ & $\beta_{1}$ & Negativo \\
\hline
\end{tabular}




\begin{tabular}{|l|l|l|l|}
\hline SP & Salario en C\$/Mes & $\beta_{2}$ & Negativo \\
\hline Edad & Edad del jefe del hogar & $\beta_{3}$ & Negativo \\
\hline TV & $\begin{array}{l}\text { Periodo de tiempo invertido mensualmente } \\
\text { en desplazarse por agua en minutos }\end{array}$ & $\beta_{4}$ & ----------------------- \\
\hline NP & Números de personas en la vivienda & $\beta_{5}$ & Positivo \\
\hline $\mathrm{P}$ & Precio por galón de agua & $\beta_{6}$ & Negativo \\
\hline
\end{tabular}

Fuente: Autores

En el modelo, las variables que justifican y representan las más importantes es el NSA (variable dependiente) y el costo (variable independiente) dentro de la metodología empleada para el estudio. Así mismo, fue necesario incluir variables socioeconómicas ${ }^{1}$ que puedan identificar de manera mejor el comportamiento del modelo.

La medida de bienestar económico que refleja el valor económico de los consumidores se puede determinar por medio del excedente del consumidor. Para esto es necesario estimar la demanda a partir de los valores medios de las variables CT, SP, edad, TV, NP y P de los consumidores, para luego poder determinar el excedente del consumidor.

Función de demanda

$$
\begin{aligned}
& N S A=\beta_{1} C T^{\beta_{2}} S P^{\beta_{3}} \operatorname{Edad}^{\beta_{4}} \operatorname{TV}^{\beta_{5}} N P^{\beta_{6}} P^{\beta_{7}} e^{u} \ldots \ldots \ldots \ldots \ldots[1]
\end{aligned}
$$

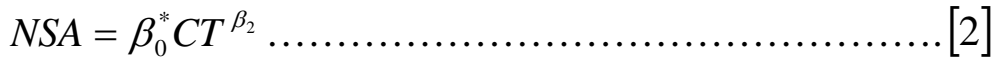

$$
\begin{aligned}
& \beta_{0}^{*}=e^{c} S P^{\beta_{3}} \operatorname{Edad}^{\beta_{4}} T^{\beta_{5}} N P^{\beta_{6}} P^{\beta_{7}} \\
& c=\ln \beta_{1}
\end{aligned}
$$

Excedente del consumidor (EC)

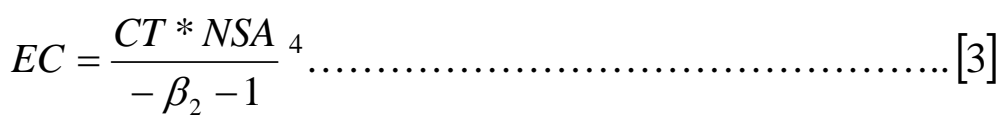

EC: Excedente del consumidor de las familias que no cuentan con el servicio de abastecimiento de agua potable por medio de ENACAL, las empresas de Matagalpa y Jinotega (AMAT y MAJIN).

El excedente del consumidor es la medida que representa el costo de la persona que realiza la actividad en obtener agua (costo), es decir, el costo en el que incurre como consecuencia del precio por el número de viajes por agua. Este valor representa una estimación adecuada de los costos en que incurre el individuo, el cual contribuye al bienestar de la familia. 


\section{Resultados}

El costo de oportunidad en el tiempo es de aproximadamente $12 \mathrm{C} \$ /$ mes y el consumo promedio de las personas que no tienen agua es de 1.7 metros cúbicos mensuales. Este resultado es muy interesante ya que al compararlo con los consumos medios de los clientes de ENACAL (ilustración 1), es 10 veces menor. Éstos consumen en promedio 5 galones o aproximadamente 20 litros por día en un hogar. El salario promedio, en base a la EMNV 2005 (INIDE, 2005a) fue de $1,805.315^{2}$ córdobas C $\$$.

La función de demanda estimada a través del método de mínimos cuadrados ordinarios (MCO) (ver salidas en Stata 10 en anexos), es la siguiente:

$$
N S A=e^{2.5575} C T^{-0.00651} S P^{-0.000016} E_{d a d}^{-0.002127} T V^{-0.0036077} N P^{0.008003} P^{0.0110594}
$$

Cuadro 7. Estimación del modelo lin-log

\begin{tabular}{|c|c|c|c|}
\hline Variables/Modelo & NSA & $P>|t|$ & Elasticidades \\
\hline Constante & 2.557531 & 0.000 & ------ \\
\hline CT & -0.0065148 & 0.000 & -0.1971933 \\
\hline $\begin{array}{c}\text { SP } \\
\text { (Sal) }\end{array}$ & -0.000016 & 0.000 & -0.0288292 \\
\hline $\begin{array}{c}\text { Edad } \\
(\mathrm{s} 2 \mathrm{p} 4 \mathrm{a})\end{array}$ & -0.002127 & 0.000 & -0.0975495 \\
\hline $\begin{array}{c}\text { TV } \\
\text { (Tminutos) }\end{array}$ & -0.0036077 & 0.000 & -0.0446136 \\
\hline $\begin{array}{c}\text { NP } \\
\text { (s1p1) }\end{array}$ & 0.008003 & 0.007 & 0.0480896 \\
\hline $\mathbf{P}$ & 0.0110594 & 0.000 & 0.1117242 \\
\hline Estadísticos & \multicolumn{3}{|c|}{$\begin{array}{l}\mathrm{R}^{2}=0.1864 \\
6,2334)=89.11 \\
\text { ber of obs }=2,341 \\
249.84 \quad \mathrm{X}^{2}=12.59\end{array}$} \\
\hline
\end{tabular}

\section{Fuente: Autores}

Para probar la bondad de ajuste del modelo se usó el test de Wald ${ }^{3}$. El valor del estadístico para el modelo fue de 249. 84, el $\mathrm{X}_{\mathrm{t}}^{2}$ tabulado con 6 grados de libertad por el número de restricciones impuesta a la hipótesis nula (el coeficiente de todas las variables incluidas en el modelo es igual a cero), por tanto se puede inferir que el ajuste del modelo en general es bueno.

Los signos son los esperados, excepto el precio por galón (P) de agua, ya que no cumple con la ley de la demanda (un aumento del precio disminuirá el número de viajes a la fuente). Esto se debe a que el precio por el bien es muy bajo (1C\$ en promedio por galón). El efecto de los coeficientes es considerado semi-elasticidad de cada una de las variables. Esto nos muestra 
que el número de viajes por agua mensual cambia en promedio según el valor proporcional de cada coeficiente, cuando cada una de las variables independientes fluctué en una unidad. Se tiene que el coeficiente del costo total (CT) es menor que cero. Esto significa que un cambio positivo en $1 \mathrm{C} \$$ en el costo del viaje implica en promedio una variación negativa del $0.65 \%$ de unidades en la cantidad demandada de viajes por agua o simplemente interpretamos las elasticidades, las cuales si el CT aumenta en 1\%, el número de viajes (NSA) disminuirá en $19 \%$.

El coeficiente del salario del jefe del hogar (SP) es menor que cero. Un aumento en 1\% del salario genera una disminución en el número de viajes del 2\%. Esto puede ocurrir ya que pueden pagar a personas que traigan el agua a la vivienda. El tamaño del jefe del hogar (NP) varía positivamente y si éste aumenta en 1\%, el número de viajes aumenta en 4\%. El resultado es lógico porque el consumo será mucho mayor.

La variable tiempo (TV) presenta un coeficiente menor que cero. Si éste aumenta en 1\%, el número de viajes disminuirá en 4\%, ya que tendrá que viajar una mayor distancia. Esto evidencia la relevancia del costo de oportunidad en el tiempo para este grupo de viviendas que no cuentan con agua por medio de la red de ENACAL, AMAT y MAJIN.

La edad presenta el signo esperado y si ésta aumenta en 1\%, el número de viajes disminuirá en 9\%. Las personas tendrán más dificultad para trasladar el agua ya sea de un río, lago, laguna, etc.

A partir de la función de demanda estimada por viajes se puede estimar el excedente del consumidor como medida de los beneficios de los hogares por contar con agua potable y los valores de costo, ecuación $\{3\}$.

$$
\mathrm{EC}=316.553 \mathrm{C} \$
$$

Una vivienda en Nicaragua tendría una ganancia en bienestar de $316.553 \mathrm{C} \$$ por mes por tener agua potable en sus viviendas por medio de tubería. La anterior medida de bienestar presenta una ventaja ante la estimación de un beneficio a partir del método de valoración contingente. La ventaja radica en que la información utilizada para la estimación de la medida de beneficios toma en cuenta las preferencias reveladas de los hogares que actualmente no acceden a la red de acueductos y alcantarillados.

Tomando como referencia el Censo 2005 (INIDE, 2005b), en Nicaragua existe 1 millón de viviendas, y en base a registros de facturación de ENACAL, existen 450 mil viviendas con agua. Al sumar los departamentos de Matagalpa y Jinotega podríamos decir que aproximadamente 500 mil viviendas no tienen agua, por tanto los beneficios sociales por llevar el agua serían de 158 millones de C\$, equivalentes a unos 7, 500,000.00 dólares por mes.

En base al EC, una vivienda pagaría hasta $14 \mathrm{C} \$$ por metro cúbico $\left(\mathrm{M}^{3}\right)$ de agua $(1,000$ litros). El resultado se puede justificar ya que por 1 galón de agua (3.8 litros) ellos pagan $1 \mathrm{C} \$$, por un barril (200 litros) $8 \mathrm{C} \$$ en promedio, lo que significa $40 \mathrm{C} \$ / \mathrm{M}^{3}$. Es importante resaltar que actualmente los precios cobrados por ENACAL (ver anexos pliego tarifario actual) en viviendas que cuentan con este servicio son mucho más bajos. 
Tendría que regularse el consumo para tomar en cuenta la restricción del ingreso y que no tiendan a caer en mora. Se ha probado que un hogar puede pagar el 10\% de su ingreso. Si se asume un salario mínimo de 2,000 C\$/Mes, estas viviendas pueden consumir unos $15 \mathrm{M}^{3}$ / Mes, lo que equivale a 9 veces más consumo cuando acceden a la red de acueductos y pasan de no tener agua potable a tenerla.

Es importante resaltar en base al pliego tarifario que una familia con categoría domiciliar, con consumos de $15 \mathrm{M}^{3} / \mathrm{Mes}$ y con medidor en su vivienda tendría una factura mensual de $98.74 \mathrm{C} \$ /$ Mes. Independientemente de si tienen alcantarillado sanitario (CVAS) o si no lo tienen, su factura es de $76.99 \mathrm{C} \$ /$ Mes en los departamentos. En Managua, los precios son diferentes.

Por tanto, una vivienda paga actualmente el $\mathrm{M}^{3}$ a $4.85 \mathrm{C} \$$ siempre y cuando consuma menos de $20 \mathrm{M}^{3}$ por mes. A esto se le tiene que sumar el cargo fijo por factura equivalente a $4.24 \mathrm{C} \$$ (ver 4 y 5 en anexos). Los precios del agua son favorables dados los ingresos del país.

\section{Conclusiones y recomendaciones}

Las formas para acceder al bien agua en una vivienda que no cuenta con el servicio son muy diversas y muchas veces se requiere realizar viajes para obtener un galón de agua para uso en el hogar.

El argumento económico es una razón necesaria y suficiente para la toma de decisiones en la gestión de políticas que ayuden a reducir la pobreza. Los resultados obtenidos en esta investigación deben ser considerados como el valor económico que representa para las personas el no contar con agua por medio de la red de ENACAL en términos de una valoración parcial que sólo considera los costos por consumo de agua y no los costos de otros valores como enfermarse por ingerir agua de baja calidad.

Los beneficios potenciales para una vivienda por tener agua serían iguales a 316.553 C\$/Mes. Si tomamos el ingreso promedio mensual del hogar 1,805.315 C\$, este valor de beneficios representa el 18\% del ingreso obtenido.

Si tomamos en cuenta las tarifas actuales y los consumos promedios en los departamentos, una vivienda con un consumo igual a $20 \mathrm{M}^{3}$ paga mensualmente $130 \mathrm{C} \$$. El excedente del consumidor equivale a 316.553 $\mathrm{C} \$ / \mathrm{Mes}$, representando una ganancia en bienestar derivada del consumo de agua potable. En las viviendas que no cuentan con este servicio, su consumo de agua les cuesta $316.553 \mathrm{C}$. El proyecto de abastecimiento de agua les aumentará el consumo 15 veces más y el gasto será mucho menor (130-200 C\$/Mes).

Para estudios futuros es necesario tomar en cuenta otros enfoques de estimación de beneficios, como valoración Cojoint, el modelo de producción salud que permitan estimar los beneficios del proyecto de agua potable y alcantarillado.

Es necesario construir el escenario base más preciso de demanda de viajes de los que no cuentan con el servicio de agua por medio de la red de ENACAL, AMAT y MAJIN. Así mismo, 
incluir las variables socioeconómicas que permitan clasificar a las viviendas en las diferentes categorías del pliego tarifario con el propósito de que se pague el costo económico del agua que en la mayoría de las viviendas está por debajo del precio de equilibrio. Los usuarios no deben pagar las pérdidas en que incurre la empresa por agua no contabilizada y por la falta de mantenimiento al acueducto en los últimos 20 años.

\section{Notas}

1 Una de las variables independientes de importancia en la metodología de costo de viaje es la del bien sustituto, que en el estudio es dificil especificar con exactitud debido a que en muchos lugares las fuentes de agua no son accesibles: -Un pozo en alguna vivienda, porque la localidad se encuentra en el cerro.

-La estatal de agua (ENACAL) no oferta el bien debido a los costos de producción elevados y comunidades con poblaciones pequeñas.

2 Este salario fue obtenido eliminando las observaciones de viviendas con acceso al agua por medio de tubería.

3 Este proceso es generalmente realizado por el contraste de razón de verosimilitud, pero debido a que no se tiene los estimadores restringido y no restringido del modelo, el más adecuado a realizar es por medio del contraste de wald debido a que sólo se precisa el estimador no restringido (Greene, 1999, p. 144).

\section{Anexo 1. Salidas usando Stata 10 del modelo lin-log}

$\begin{array}{rrrr}\text { Source | } & \text { SS } & \text { df } & \text { MS } \\ \text { Model | } 90.1877039 & 6 & 15.031284 \\ \text { Residual | } & 393.710911 & 2334 & .168685052 \\ \text { Total | } 483.898615 & 2340 & .20679428\end{array}$

$\begin{array}{lrr}\text { Number of obs } & 2341 \\ \mathrm{~F}(6,2334) & =89.11 \\ \text { Prob }>\mathrm{F} & =0.0000 \\ \text { R-squared } & 0.1864 \\ \text { Adj R-squared } & =0.1843 \\ \text { Root MSE } & =.41071\end{array}$

\begin{tabular}{|c|c|c|c|c|c|c|}
\hline INSA I & Coef. & Std. Err. & $\mathrm{t}$ & $P>|t|$ & [95\% Conf. & Interval] \\
\hline$-\cdots-$ & 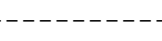 & $-\ldots$ & ----- & - - - - & ------- & 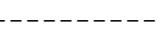 \\
\hline s1p1 | & .008003 & .0029485 & 2.71 & 0.007 & .0022211 & .0137849 \\
\hline CT । & -.0065148 & .0003297 & -19.76 & $\odot .000$ & - . .0071614 & -.0058682 \\
\hline Tminutos | & - . .0०36077 & $.0 \odot \odot 6172$ & -5.85 & $\odot . \odot \odot \odot$ & - . .๑4818 & - . . \\
\hline Sal | & - . .๑๑९16 & $2.28 e-06$ & -7.01 & $\odot .0 \odot \odot$ & - . .๑९९2०4 & - . . \\
\hline P I & .0110594 & .0005848 & 18.91 & 0.000 & .0099125 & .0122063 \\
\hline $\mathrm{s} 2 \mathrm{p} 4 \mathrm{a} \mid$ & -.002127 & .0005414 & -3.93 & 0.000 & - . .0๑31886 & -.0010653 \\
\hline _cons | & 2.557531 & .0330898 & 77.29 & 0.000 & 2.492643 & 2.622419 \\
\hline
\end{tabular}


Anexo 2. Elasticidades del modelo lin-log

Elasticities after regress

$y=$ Fitted values (predict)

\begin{tabular}{|c|c|c|c|c|c|c|c|}
\hline variable | & $\mathrm{dy} / \mathrm{ex}$ & Std. Err. & z & $P>|z|$ & $95 \%$ & C.I. & $X$ \\
\hline \multicolumn{8}{|c|}{ 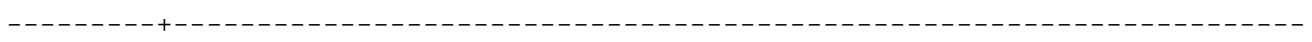 } \\
\hline s1p1 | & .0480896 & .01772 & 2.71 & $\odot . \odot \odot 7$ & .013364 & .082815 & $6.0 \odot 897$ \\
\hline СТ । & - . 1971933 & .00998 & -19.76 & $\odot .00 \odot$ & -.216753 & -.177633 & 30.2685 \\
\hline Tminutos | & - . . 0446136 & $.0 \odot 763$ & -5.85 & $\odot .000$ & - . . 059572 & - . .029655 & 12.3661 \\
\hline Sal | & - . . 0288292 & .00411 & -7.01 & $\odot .0 \odot \odot$ & - . .036894 & - . . 020764 & 1804.55 \\
\hline$P \quad \mid$ & .1117242 & .00591 & 18.91 & 0.000 & .100144 & .123304 & 10.1022 \\
\hline s2p4a | & - . . 0975495 & .02483 & -3.93 & $\odot . \odot \odot \odot$ & -.146216 & -.048882 & 45.8633 \\
\hline
\end{tabular}

\section{Anexo 3. Pliego tarifario aplicado a los usuarios de ENACAL}

Managua, Veintinueve de Febrero del Dos Mil Ocho.

TARIFAS INDEXADAS A JUNIO DE zOOE

EMPRESA NICARAGUENSE DE ACUEDUCTOS Y ALCANTARILLAOOS (ENACAL)

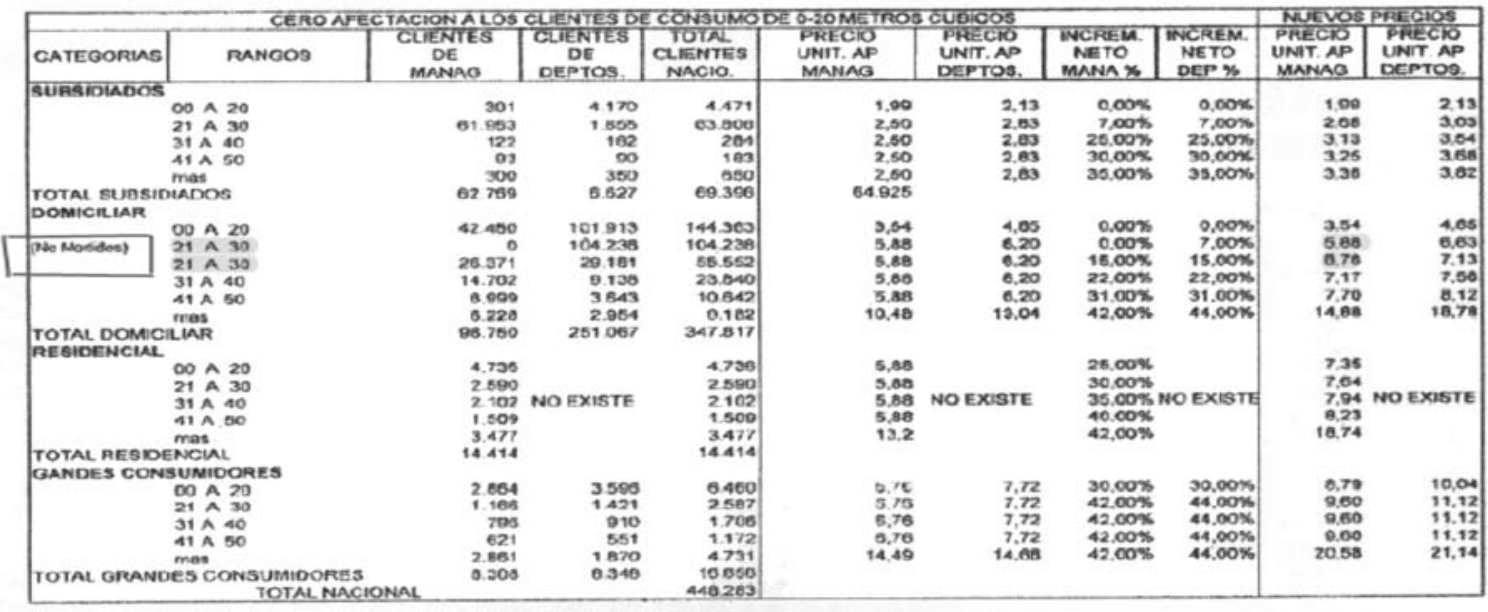

ENACAL: EL 95\% DE LAS TARIFAS CONTINÚUN SUBSIDIADAS.

Anexo 4. Pliego tarifario aplicado a los usuarios de ENACAL en la categoría domiciliar en los departamentos

\begin{tabular}{|c|c|c|c|}
\hline Rangos de Consumo en M3 & CF- C\$ & $\mathrm{CVAP}-\mathrm{C} \$ / \mathrm{M}^{3}$ & CVAS- $\mathrm{C} \$ / \mathrm{M}^{3}$ \\
\hline $0-20$ & \multirow{5}{*}{4.24} & 4.85 & 1.45 \\
\hline $21-30$ & & 7.13 & 1.78 \\
\hline $31-40$ & & 7.56 & 1.78 \\
\hline $40-50$ & & 8.12 & 1.78 \\
\hline Mas & & 18.78 & 3.57 \\
\hline
\end{tabular}


CF: Cargo fijo por cliente aplicado una vez por emisión

CVAP: Cargo variable de agua potable en C\$

CVAS: Cargo variable de alcantarillado

Anexo 5. Ejemplo de aplicación del pliego tarifario en una vivienda con un consumo mensual de $15 \mathrm{M}^{3}$

\begin{tabular}{|c|c|c|c|c|}
\hline $\begin{array}{l}\text { Rangos de Consumo } \\
\text { en M3 }\end{array}$ & $\begin{array}{c}\text { Consumo } \\
{\text { en } M^{3}}^{2}\end{array}$ & $\mathrm{CF}-\mathrm{C} \$$ & CVAP- $\mathrm{C} \$ / \mathrm{M}^{3}$ & CVAS- $\mathrm{C} \$ / \mathrm{M}^{3}$ \\
\hline $0-20$ & 15 & & $15 * 4.85=72.75$ & $15 * 1.45=21.75$ \\
\hline \multicolumn{4}{|l|}{$21-30$} & \\
\hline \multicolumn{4}{|l|}{$31-40$} & \\
\hline \multicolumn{4}{|l|}{$40-50$} & \\
\hline \multicolumn{4}{|l|}{ Mas } & \\
\hline Total facturación & & & \multicolumn{2}{|c|}{$4.24+72.75+21.75=98.75 \mathrm{C} \$$} \\
\hline
\end{tabular}

Fuente: Autores

Anexo 6. Ejemplo de aplicación del pliego tarifario en una vivienda con un consumo mensual de $30 \mathrm{M}^{3}$

\begin{tabular}{|c|c|c|c|c|}
\hline $\begin{array}{l}\text { Rangos de Consumo } \\
\text { en M3 }\end{array}$ & $\begin{array}{c}\text { Consumo } \\
{\text { en } \mathrm{M}^{3}}^{2} \\
\end{array}$ & CF- C\$ & CVAP- $\mathrm{C} \$ / \mathrm{M}^{3}$ & CVAS- C\$/M $\mathrm{M}^{3}$ \\
\hline $0-20$ & 30 & \multirow{5}{*}{4.24} & $20 * 4.85=97$ & $20 * 1.45=29$ \\
\hline $21-30$ & & & $10 * 7.13=71.3$ & $10 * 1.78=17.8$ \\
\hline \multicolumn{2}{|l|}{$31-40$} & & & \\
\hline \multicolumn{4}{|l|}{$40-50$} & \\
\hline \multicolumn{4}{|l|}{ Mas } & \\
\hline Total facturación & & & $4.24+97+29+71$ & $=219.34 \mathrm{C} \$$ \\
\hline
\end{tabular}

Fuente: Autores 


\section{Referencias bibliográficas}

Avendaño, R. (2004). Estudio económico para la definición de cánones para el uso y aprovechamiento del recurso hídrico para Nicaragua. Global Water Partnership. Recuperado el 10 de febrero de 2010 de http://www.docstoc.com/docs/3271966/Enfoquesmetodol\%C3\%B3gicos-y-estimaciones-del-valor-econ\%C3\%B3mico-del-agua-

Banco Central de Nicaragua [BCN]. (2010).Anuario de Estadísticas Mensuales. Recuperado el 15 de enero de 2010 de http://www.ben.gob.ni

Bockstael, N. \& McConnell, K. (1983). Welfare Measurement in the Household Production Framework. American Economic Review. (73), 806-814.

Call, S. \& Holahan, W. (1983). Microeconomía. México D.F.: Grupo Editorial Iberoamericana S. A. de C. V.

Empresa de Acueductos y Alcantarillados [ENACAL]. (2007 y 2008). Consumo y producción de agua por departamento. Base de datos en soporte CD. Managua: ENACAL.

Greene, W. H. (1999). Análisis Econométrico. (3era Ed.). Madrid: Prentice Hall.

Hanemmann, M. (1998). Determinants of Urban Water Use. En D. Bauman, J. Boland \& W. M. Hanemann, (eds.). Urban Water Demand Management and Planning. New York: Mcgraw Hill Book Co., pp 31-52.

Instituto Nacional de Información de Desarrollo [INIDE]. (2005a). Encuesta de Medición del Nivel de Vida-EMNV. Base de datos en soporte CD. Managua: INIDE.

Instituto Nacional de Información de Desarrollo [INIDE]. (2005b). VIII Censo de Población y IV de Vivienda. Base de datos en soporte CD. Managua: INIDE.

Instituto Nacional de Información y Desarrollo [INIDE]. (2001). III Censo Agropecuario. Base de datos en soporte CD. Managua: INIDE.

Instituto Nicaragüense de Acueductos y Alcantarillados [INAA]. (2008). Ente Regulador. Resolución No. CD-RE-037-2008, Managua: INAA.

Jouravlev. A. (2008). Situación en la Región y Casos Nacionales: Gestión y Servicio. Comisión Económica para América Latina y el Caribe (CEPAL). Presentación realizada en Santiago de Chile. Sala Anfiteatro Fernando Sajnzylber. Oficina CEPAL, Av. Dag Hammarskjold 3477, Vitacura, Santiago de Chile. 10 de abril de 2007.

Mendieta, J. \& Caraballo, L. (2005). Economía de la contaminación y de degradación ambiental. Sancristóbal-Venezuela: Fondo Editorial Nuevo Tiempo.

Reddy, R. V. (1999). Quenching the Thirst: The Cost of Water in Fragile Environments. Institute of Social Studies Oxford OX4 1JF, UK. Recuperado el 15 de enero de $2010 \mathrm{dehttp} / / /$ www3.interscience.wiley.com/journal/119066145/abstract?CRETRY $=1 \&$ SRETRY $=0$ Romero, C. (1997) Economía de los Recursos Naturales y Ambientales. Madrid: Alianza Editorial, S.A.

Smith, V. \& Kaoru, Y. (1990) Signals or Noise? Explaining the Variation in Recreation Benefit Estimates. American Journal of Agricultural Economics, 72 (2), 419-433.

Strand, J. \& Walter, I. (2003) Water Allocation and Demand in Central America: Analysis of Tap and Non-Tap Water Demand in 17 Central American Cities. University of Oslo. Tegucigalpa Honduras. Recuperado el 12 de enero de 2010 de http://www.sciencedirect. $\mathrm{com} / \mathrm{science} ? \mathrm{ob}=$ MImg\& imagekey $=$ B6VFJ-4KPFKHG-1-1\&_cdi $=6012 \&$ user $=10 \&$ $\mathrm{pii}=\mathrm{S} 09287 \overline{6} 55060003 \overline{3} 9 \&$ \& orig $=$ search\&_coverDate $=09 \% 2 \mathrm{~F} 30 \% \overline{\mathrm{F}} 2007 \mathrm{\&}_{-}^{-}$ $\mathrm{sk}=999709996 \&$ view $=\mathrm{c} \& \mathrm{wch} \bar{p}=\mathrm{dGLbVlb}-\mathrm{zSkzk} \& \mathrm{md} 5=91719 \mathrm{~d} 4 \mathrm{f} 4 \mathrm{a} 35 \mathrm{f} 786002 \mathrm{~d} 5 \mathrm{f} 60 \mathrm{~b} 9 \mathrm{a} \overline{4}$ 9b96\&ie=/sdarticle.pdf 
Torrado Briceño, E. A. (2001) Efectos políticas de precios y programas de conservación sobre el consumo del agua. Caso Bogotá. Tesis para optar al título de Master en Economía del Medio Ambiente y los Recursos Naturales. Facultad de Economía, Universidad de los Andes, Colombia.

Uribe, E., Mendieta, J., Jaime, J. \& Carriazo, F. (2003). Introducción a la Valoración Ambiental de Estudios de Caso. Universidad de Los Andes. Facultad de Economía. CEDE: Ediciones Uniandes.

Víquez, A. Q. (2005). La situación del agua en Nicaragua. Menschen Recht. WASSER. Recuperado el 12 de marzo de 2010 de http://www.simas.org.ni/_simasnoticia/ Situacion $\% 20$ del $\% 20$ agua $\% 20$ en $\% 20$ Nicaragua.pdf 